\title{
The DIMINUTO gene of Arabidopsis is involved in regulating cell elongation
}

\author{
Taku Takahashi, Alexander Gasch, Naoko Nishizawa, ${ }^{1}$ and Nam-Hai Chua ${ }^{2}$ \\ Laboratory of Plant Molecular Biology, The Rockefeller University, New York, New York 10021-6399 USA; ${ }^{1}$ Faculty of \\ Agriculture, University of Tokyo, Bunkyo-ku, Tokyo 113, Japan
}

\begin{abstract}
We have isolated a recessive mutation named diminuto (dim) from T-DNA transformed lines of Arabidopsis thaliana. Under normal growth conditions, the dim mutant has very short hypocotyls, petioles, stems, and roots because of the reduced size of cells along the longitudinal axes of these organs. In addition, dim results in the development of open cotyledons and primary leaves in dark-grown seedlings. The gene for DIM was cloned by T-DNA tagging. DIM encodes a novel protein of 561 amino acids that possesses bipartite sequence domains characteristic of nuclear localization signals. Molecular and physiological studies indicate that the loss-of-function mutant allele does not abolish the response of seedlings to light or phytohormones, although the inhibitory effect of light on hypocotyl elongation is greater in the mutant than in wild type. Moreover, the $\operatorname{dim}$ mutation affects the expression of a $\beta$-tubulin gene, TUB1, which is thought to be important for plant cell growth. Our results suggest that the DIM gene product plays a critical role in the general process of plant cell elongation.
\end{abstract}

[Key Words: Arabidopsis mutant; T-DNA tagging; cell elongation; tubulin genes; nuclear localization signals]

Received October 13, 1994; revised version accepted November 29, 1994.

The specific pattern and morphology of a given multicellular organism is attributable, in part, to mechanisms that regulate the shape, size, and number of its cells. Unlike animal cells, whose differentiation is often irreversible, almost all living cells of higher plants possess the potential to develop into an entire plant, that is, they are totipotent (Steeves and Sussex 1989). The plasticity inherent in plant cells suggests that growth and differentiation in higher plants may be dependent to a great extent on external environmental stimuli.

One of the most important external signals is light. The effect of light on plant development (photomorphogenesis) has been studied extensively (for review, see Deng 1994). Recent experiments have provided insight into the signal transduction pathways that regulate the expression of light-responsive genes (e.g., Bowler et al. 1994). In contrast, little is known about how light signals inhibit hypocotyl cell elongation and induce cellular differentiation in the hypocotyl and cotyledons. No target molecules of light signals that control cell growth have been identified. The photomorphogenic pathways that affect plant cell growth are difficult to analyze because they overlap with phytohormone signal transduction pathways. For instance, the phototropic response of a growing shoot tip is mediated by auxin. Moreover, the elongated internode (ein) mutant of Brassica, which is known to have a defect in photoperception also has an

${ }^{2}$ Corresponding author. increased production of gibberellin (GA) (Rood et al. 1990; Devlin et al. 1992). In these examples, auxin or GA apparently plays a role in stimulating cell elongation. Chory et al. (1994) have also reported that cytokinins act in the light-dependent expansion of cotyledons and leaves.

The final shape of plant organs is a consequence of cell division and cell expansion. In meristematic tissues, cell division is followed by cell expansion, which determines the shape of individual cells. Among the known components involved in determining the direction of cell expansion are plasma membrane-associated cortical microtubules. It is well-known that longitudinal growth or elongation is associated with the transverse arrangement of cortical microtubules (Fosket and Morejohn 1992; Shibaoka 1994), which are thought to direct the orientation of cellulose microfibrils in the cell wall. In turn, the growth rate of plant cells, which is controlled by the extensibility of the cell wall and the turgor pressure inside the cell (Cosgrove 1986), is considered to be important for microtubule rearrangement. In spite of numerous physiological studies focusing on the molecular properties of these structural components (for review, see Carpita and Gibeaut 1993; Shibaoka 1994), it is largely unknown how light signals and phytohormones regulate the process of cell growth. To facilitate a more detailed investigation of the control of plant cell morphogenesis, the basic regulatory molecules that determine the amount and direction of cell expansion must be identified. 
We are taking a genetic approach to identify such critical components. In this report we describe the characterization of a T-DNA-tagged mutant of Arabidopsis, named diminuto (dim). Cell elongation in every organ of the dim mutant is greatly reduced. We cloned the DIM gene and found that it encodes a novel protein with no sequence similarity to known proteins. The mutation appears to affect the expression of a tubulin gene, TUB1. Based on the phenotype of the mutant plants, we discuss the significance of the DIM protein during plant cell growth. The high similarity between the DIM protein and an open reading frame from a human-expressed sequence-tag clone suggests that DIM and the predicted human protein may share a conserved function.

\section{Results}

Phenotype of the dim mutant

The mutant used in this study was originally identified as a short hypocotyl (hy) mutant in transgenic Arabidopsis lines produced in our laboratory. The mutant was named $\operatorname{dim}$ for its reduced stature. Figure 1A illustrates the dim phenotype in young seedlings grown under white light. Compared with wild-type seedlings, dim seedlings have much shorter hypocotyls, petioles, and roots. In addition, leaves of dim are round, curly, and dark green in color. In adult flowering plants, dim shows extremely short inflorescences and small flowers (Fig. 1B), and fertility is severely reduced. It is important to note that the effect of the dim mutation on leaf morphology is limited sharply to the longitudinal direction of the leaf (Fig. 1C). Observations using light microscopy revealed that these morphological defects are primarily attributable to a reduction in cell size along the longitudinal axes of organs, as shown in Figures 1, E and G, and 2B. In mature leaves, dim mesophyll cells are tighter and more expanded in the dorsal-ventral axis of the leaf than wild-type cells, thereby leading to an increase in the thickness of the leaf (cf. Fig. 1, D and E). In addition to the morphological abnormalities, dim exhibits slightly delayed flowering. Table 1 summarizes the phenotypic characteristics of light-grown dim plants. The darkgreen color of dim leaves is probably attributable, at least in part, to the increased density of chloroplasts per leaf area, as the size of dim cells is smaller in the long axis of
Figure 1. Phenotypes of light-grown wild-type and dim Arabidopsis plants. $|A|$ Ten-day-old wild-type (WT) and dim seedlings. (B) A 30-day-old dim plant. $(C)$ Leaf morphology of wild-type (top) and dim (bottom) plants. $(D, E)$ Longitudinal sections of the first true leaves of wild-type $(D)$ and $\operatorname{dim}(E)$ plants examined by light microscopy. $(F, G)$ Longitudinal sections of mature inflorescences (the lowermost internode of 30-day-plants) of wild-type $(F)$ and $\operatorname{dim}(G)$ plants. Bars, $10 \mathrm{~mm}$ for $B, 50$ $\mu \mathrm{m}$ for $D$, and $E$, and $100 \mu \mathrm{m}$ for $F$ and $G$.
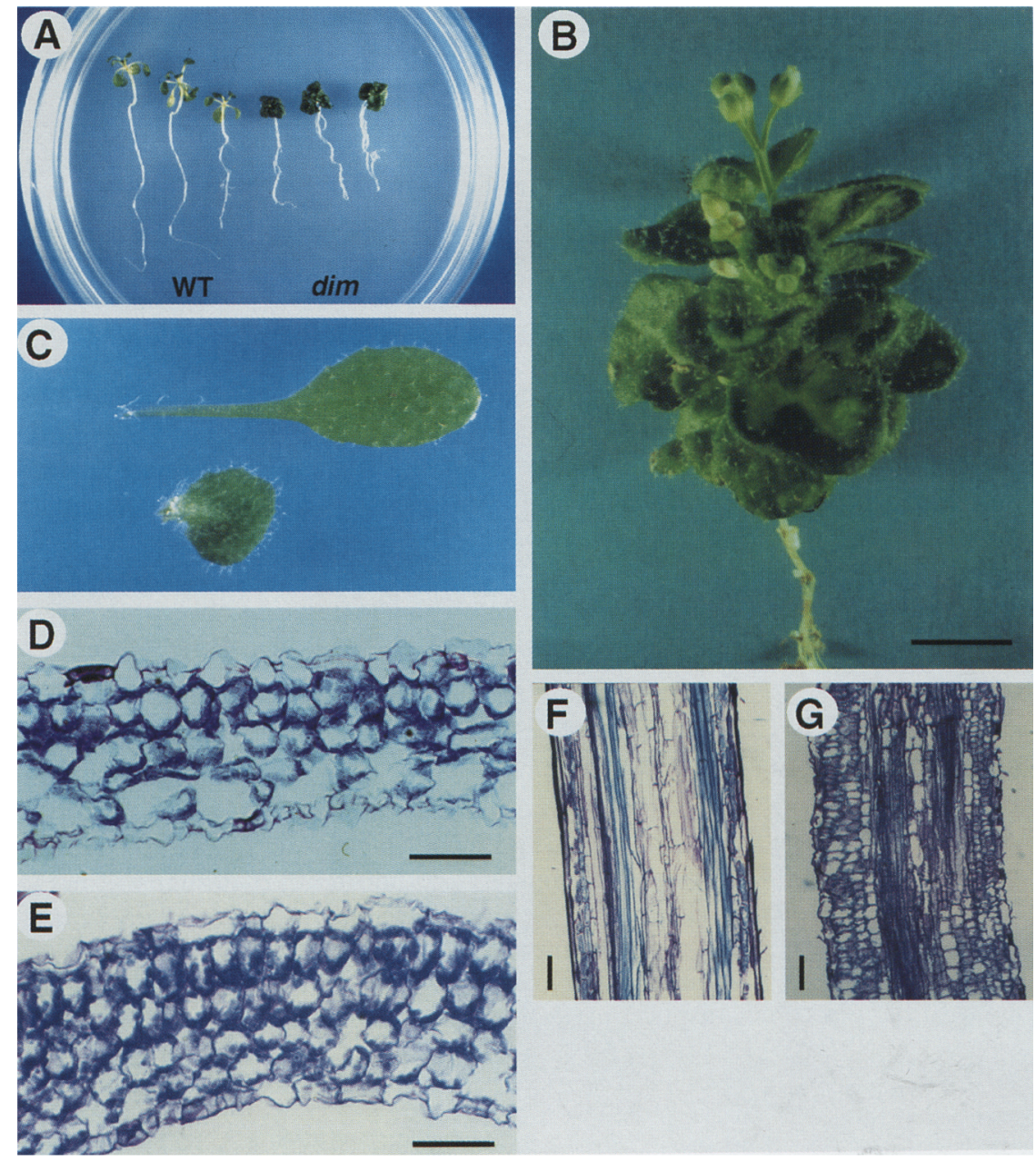
Table 1. Growth features of light-grown wild-type and dim plants

\begin{tabular}{lcc}
\hline & Wild type & dim \\
\hline 15 days & & \\
$\quad$ length of main root $(\mathrm{cm})$ & $2.6 \pm 0.4$ & $1.4 \pm 0.3$ \\
length of hypocotyl $(\mathrm{mm})$ & $3.8 \pm 0.4$ & $0.8 \pm 0.2$ \\
petiole/blade ratio & $1.1 \pm 0.1$ & $0.3 \pm 0.1$ \\
number of chloroplasts per cell & $50 \pm 14$ & $56 \pm 13$ \\
chlorphyll $a / b$ ratio & $2.36 \pm 0.20$ & $2.24 \pm 0.20$ \\
40 days & $36.5 \pm 5.5$ & $3.7 \pm 0.5$ \\
height $(\mathrm{cm})$ & $5.1 \pm 0.7$ & $2.6 \pm 0.2$ \\
rosette diameter $(\mathrm{cm})$ & $21 \pm 3$ & $25 \pm 4$ \\
Days to flower &
\end{tabular}

Plants were grown in a 16 -hr light/8-hr dark cycle. Data represent mean ( \pm S.E.) of 5 or 50 (for chloroplast number) samples. Measurements of the number of chloroplasts were made on leaf mesophyll protoplasts.

leaves, yet these cells appear to contain about the same number of chloroplasts as wild-type cells. Moreover, we found no major difference in the chlorophyll $a / b$ ratio between wild-type and dim plants (Table 1).

When grown in the dark, dim seedlings develop short hypocotyls, no apical hooks, open cotyledons, and primary leaf buds, in contrast to wild-type seedlings that have elongated hypocotyls with closed cotyledons (Figs. $2 \mathrm{C}, \mathrm{D}$ and $3 \mathrm{~B})$. The development of trichomes can be also identified on primary leaves of dark-grown dim seedlings (Fig. 2D). The dark phenotype of dim is similar to that of a series of photomorphogenic mutants (e.g., $\operatorname{det} 2$ ) displaying a "de-etiolated" phenotype (Chory 1992). After $>10$ days of dark growth, however, dim does not exhibit further differentiation of true leaves, as is the case in the det 1 (Pepper et al. 1994) and copl mutants (McNellis et al. 1994). We also examined dim plastid morphology by transmission electron microscopy (Fig. 2E-H). As illustrated in Figure 2F, dim has normal etioplasts with typical prolamellar bodies in dark-grown cotyledons. When grown in the light, dim seedlings contain regular lensshape chloroplasts with grana stacks. Interestingly, these dim chloroplasts accumulate many large starch grains (Fig. 2H).

\section{Responses to light and plant hormones}

To determine whether the de-etiolated morphology of $\operatorname{dim}$ is associated with an elevated expression of lightinducible genes, we performed Northern hybridizations using RNAs from wild-type and dim 10-day-old seedlings. We found only low basal levels of expression of nuclear-encoded genes for light-harvesting chlorophyll $a / b$-binding protein $(C A B)$ and chalcone synthase $(C H S)$, and of a chloroplast-encoded gene for the large subunit of ribulose bisphosphate carboxylase/oxygenase $(R B C L)$ in dark-grown wild-type and dim seedlings (Fig. 3A). The relative abundance of these transcripts in light-grown seedlings was also unaffected by the dim mutation. To assess the possibility that the dim phenotype is attributable to a defect in photomorphogenic pathways mediated by phytochrome or blue light receptors, we further investigated the effect of light on the growth of dim. Figure 3B shows the hypocotyl length of 10-day-old seedlings grown under various light conditions. It is clear that the dim mutant responds to all light treatments examined, by having a shorter hypocotyl than in the dark. In wild-type Arabidopsis seedlings, inhibition of hypocotyl elongation in continuous red or blue light is substantially less than in white light (Liscum and Hangarter 1991; Parks and Quail 1993). In our study with wild-type seedlings, far-red light was also less effective than white light because of the low fluence rate of irradiation used (McCormac et al. 1993). The effects of these light regimes on dim are qualitatively similar to those on wild type. In these experiments the efficiency of seed germination was also not influenced by the dim mutation. These data suggest that the DIM gene product does not play a direct role in light signaling pathways.
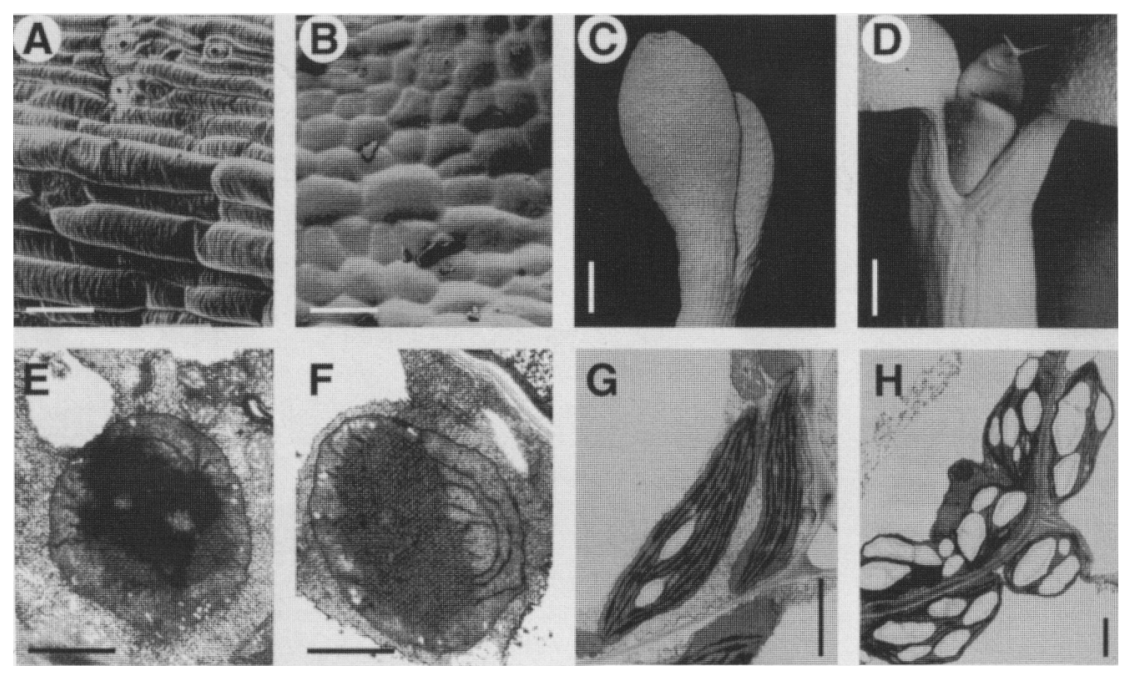

Figure 2. Electron microscopic views of wild-type and dim seedlings. $(A, B)$ Development of petiole epidermal cells. Trueleaf petioles of 10-day-old light-grown wildtype $(A)$ and $\operatorname{dim}\langle B\}$ seedlings were examined by scanning electron microscopy. $(C, D)$ Morphology of dark-grown seedlings. Only the top portions of 10-day-old wildtype $(C)$ and $\operatorname{dim}(D)$ seedlings are shown. Trichomes can be detected on the primary leaves of the dim mutant in $D .(E-H)$ Plastid development in 10-day-old dark- and light-grown seedlings. Etioplasts of darkgrown wild-type $(E)$ and $\operatorname{dim}(F)$ cotyledons, and chloroplasts of light-grown wild-type $(G)$ and $\operatorname{dim}(H)$ cotyledons are shown. Bars, $5 \mu \mathrm{m}$ for $A$ and $B, 200 \mu \mathrm{m}$ for $C$ and $D, 0.5$ $\mu \mathrm{m}$ for $E$ and $F$, and $1 \mu \mathrm{m}$ for $G$ and $H$. 
Takahashi et al.

A

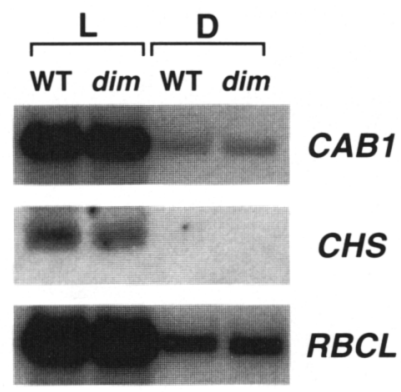

B

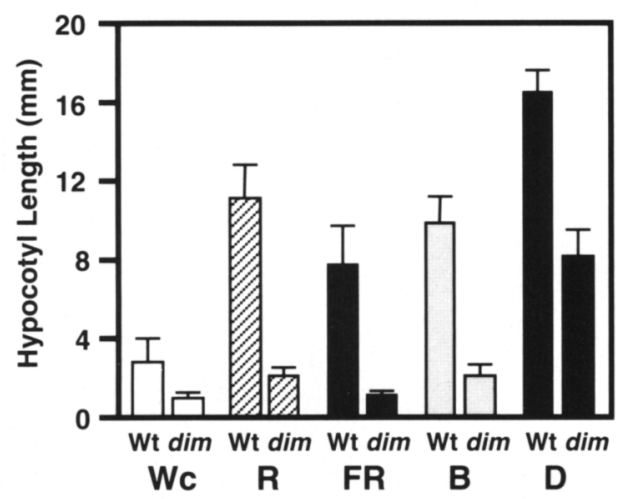

Figure 3. Light response of the dim mutant. $(A)$ Northern analysis of mRNAs for light-inducible genes. Total RNA (10 $\mu \mathrm{g})$ isolated from 10-day-old light-grown (L) and dark-grown (D) seedlings of wild-type (WT) and dim mutants was hybridized with probes for $C A B 1, C H S$, and $R B C L$, as described in Materials and methods. $(B)$ Light-regulated hypocotyl elongation in the wild-type and dim mutant seedlings. Seeds of wild-type (Wt, Columbia) and dim were sown onto agar plates and kept in the dark at $4^{\circ} \mathrm{C}$ for 2 days before transferring them to light chambers. Seedlings were grown for 10 days under continuous white (Wc, $35 \mu \mathrm{moles} / \mathrm{m}^{2}$ per sec), red $\left(\mathrm{R}, 12 \mu \mathrm{moles} / \mathrm{m}^{2}\right.$ per sec), far-red (FR, $5 \mu$ moles $/ \mathrm{m}^{2}$ per sec), or blue (B, $10 \mu \mathrm{moles} / \mathrm{m}^{2}$ per sec) light or in complete darkness (D). The mean hypocotyl length is plotted for 10 seedlings from each light regime. Bars, 1 S.E.M.

Plant growth regulators (phytohormones) play a central role in many aspects of plant development. A number of Arabidopsis mutants with altered responses to exogenously applied hormones have been isolated and shown to have aberrant morphology (for review, see Finkelstein et al. 1987). Therefore, we examined whether the dim phenotype is caused by a mutation in the hormone biosynthesis or responsive pathways. Hypocotyl sections from 5-day-old dark-grown seedlings were treated for $24 \mathrm{hr}$ with Murashige-Skoog (MS) solutions containing auxin $\left[10^{-5} \mathrm{M}\right.$ indole-3-acetic acid (IAA)], GA $\left(10^{-4} \mathrm{M} \mathrm{GA}_{3}\right)$, or cytokinin $\left[10^{-5} \mathrm{M}\right.$ 2-isopentenyladenine (2-iP)]. Under our conditions, treatment with $\mathrm{GA}_{3}$, and more effectively with $\mathrm{GA}_{3}$ in combination with IAA, enhanced hypocotyl elongation both in wild-type and dim hypocotyls. Spraying GA on intact mutant seedlings failed to restore the phenotype to normal, although main stem growth and fertility were slightly increased.
We also tested the sensitivity of mutant roots to high concentrations of IAA and 2-iP. No difference between dim and wild-type plants was observed in these experiments (data not shown). dim mutant roots did not appear to be deficient in gravitropic response, which may be hormonally regulated. From these results, we conclude that DIM is unlikely to function in the biosynthesis or signaling of phytohormones.

\section{Mapping and cloning of the DIM gene}

Genetic segregation data have indicated that dim represents a single recessive mutation and that the phenotype is tightly linked to the T-DNA insertion (data not shown). The genome map location of the DIM locus was determined by CAPS (codominant cleaved amplified polymorphic sequence) analysis (Konieczny and Ausubel 1993). Since our initial mapping data established that $D I M$ is on chromosome 3 , we focused our search for markers on chromosome 3 . An $\mathrm{F}_{2}$ population from the cross between $\operatorname{dim}$ (ecotype $\mathrm{C} 24$ ) and wild-type Columbia was scored with GAPC, GAPA, and GL1 markers. Figure $4 \mathrm{~A}$ summarizes the data obtained from $118 \mathrm{~F}_{2}$ homozygous dim plants ( 236 chromatids). The DIM gene lies $\sim 7.6 \mathrm{cM}$ from GAPC.

To investigate the molecular function of the DIM gene in plant growth, we cloned the gene. A genomic DNA fragment adjacent to the T-DNA was isolated by an inverse PCR strategy (see Materials and methods) and used as a probe to screen a wild-type Arabidopsis genomic
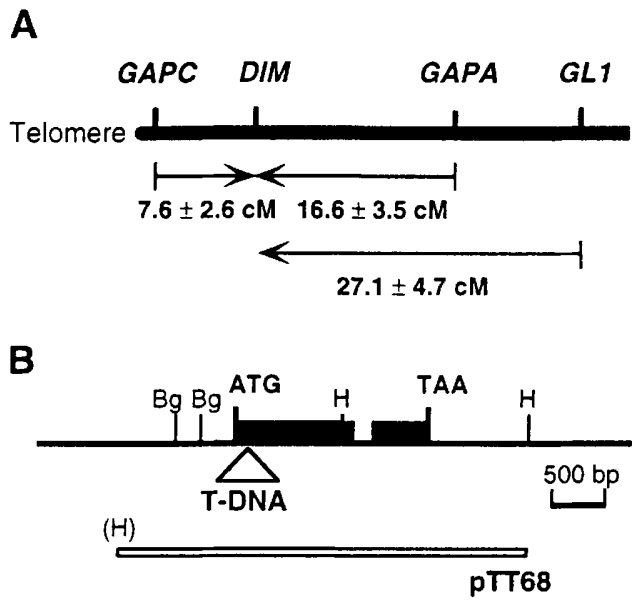

Figure 4. Genomic map and organization of the DIM gene. $|A|$ Map position of the DIM locus relative to CAPS markers. The genomic region shown corresponds to the top of chromosome 3 . Map distances (in $\mathrm{cM}$ ) were calculated from the analysis of 118 plants of an $F_{2}$ population, using the Kosambi mapping function as described by Koornneef and Stam (1992). (B) Structure of the $D I M$ gene. Solid bars represent the protein-coding regions. $(\Delta)$ The T-DNA insertion site in the dim allele. $(\mathrm{H})$ HindIII; $(\mathrm{Bg})$ $B g l I I$. The open bar represents a 4-kb HindIII fragment used to generate the plant transformation vector pTT68 for the complementation analysis. The HindIII site upstream of the DIM gene in pTT68 was artificially created by PCR for the cloning. 
library. The transcribed region interrupted by the T-DNA insertion was deduced from a comparison of Northern blots of RNAs from wild-type and mutant plants probed with subcloned genomic fragments. Two cDNA clones derived from the DIM mRNA were isolated from an Arabidopsis aerial part cDNA library (Gasch et al. 1990). One of these clones contained a 2.0$\mathrm{kb}$ cDNA insert corresponding to almost the full length of the DIM transcript. DNA sequencing of these clones revealed a long open reading frame of $1683 \mathrm{bp}$. The first methionine residue was confirmed by the presence of an in-frame stop codon upstream of the predicted start codon. Figure 4B shows the genomic structure of DIM. The DIM gene contains one intron of $97 \mathrm{bp}$. The T-DNA insertion in dim was found to disrupt the first exon of the gene.

\section{Complementation analysis}

To determine whether this gene was actually DIM, we tested the ability of a wild-type genomic DNA fragment to rescue the dim mutant phenotype. The DNA fragment covers the entire protein-coding sequence plus $\sim 1$ $\mathrm{kb}$ each of the flanking $5^{\prime}$ and $3^{\prime}$ regions /clone pTT68 in Fig. 4B). We were unable to regenerate plants from homozygous dim root callus. Therefore, wild-type plants were transformed with pTT68, using a hygromycin resistance marker, and the resulting transgenic plants were crossed with heterozygous dim mutants, which carry a kanamycin resistance marker in the T-DNA. $F_{1}$ plants that were resistant to both kanamycin and hygromycin were allowed to self-pollinate. At least two parental lines never segregated for any plants displaying the dim-like phenotype in the $F_{2}$ progeny plants, which were grown on media containing both kanamycin and hygromycin (data not shown). Interestingly, one line gave rise to $F_{1}$ plants with a dim-like phenotype in the presence of both kanamycin and hygromycin. Because these $F_{1}$ individuals are putatitvely heterozygous for both the dim mutation and the newly introduced wild-type DNA, the phe- notype might be attributed to cosuppression of the endogenous DIM gene by the introduced DIM transgene (Jorgensen 1990). On the basis of these results, we conclude that the cloned gene does correspond to the DIM locus.

\section{Sequence of the DIM protein}

DIM encodes a protein of 561 amino acids with a calculated molecular mass of $65.4 \mathrm{kD}$ (Fig. 5A). The DIM amino acid sequence was compared with sequences available in the GenBank data base. A region of 255 amino acids in the middle of the DIM protein shares strikingly high similarity (51\% identical) to RSC390, a predicted human protein with unknown function, whose cDNA was randomly cloned from a myoblast cell cDNA library (GenBank accession number D13643). The length of the human protein is much shorter than that of DIM (Fig. 5B). DIM also contains putative bipartite motifs of basic residues (Lys or Arg), characteristic of nuclear localization signals (NLSs). The DIM protein contains three such motifs, two at the amino terminus and one at the carboxyl terminus. Figure $5 \mathrm{C}$ shows the alignment of the putative NLSs present in DIM with those in other nuclear proteins. A consensus motif has been defined as a combination of two adjacent basic amino acids, a spacer region of $\sim 10$ residues, and at least 3 basic residues of the next 5 amino acids (Robbins et al. 1991). These motifs are absent in the human homolog of DIM. Potential N-linked glycosylation sites are identified at positions 147 and 278, although they are not conserved in the homologous domain of the human protein. A hydropathy plot of DIM revealed a hydrophilic region at the amino-terminal end (amino acids 1-20), followed by a hydrophobic region from amino acids $21-44$. The significance of these stretches remains to be elucidated.

\section{DIM is constitutively expressed in Arabidopsis plants}

We examined expression patterns of the DIM gene by Northern blot analysis. RNA samples from light- and
A

1 MSDLOTPLVR PKRKKTWVDY FVKFRWIIVI FIVLPFSATF 41 YFLIYLGDMN SESKSFEKRQ KEHDENVKKV IKRLKKGRDAS 81 KDGLVCTARK PWIAVGMRNV DYKRARHFEV DLGEFRNILE 121 INKEKMTARV EPLVNMGQIS RATVPMNLSL AVVAELDDLT 161 VGGLINGYGI EGSSHIYGLF ADTVEAYEIV IAGGELVRAT 201 RDNEYSDLYY AIPWSOGTLG LLVAAEIRLI KVKEYMRITY 241 IPVKGDLOAL AOGYIDSFAP KDGDKSKIPD FVEGMVYNPT 281 EGGMMVGTYA SKEEAKKKGN KINNVGWWFK PWFYQHAQTA 321 LKKGQFVVYI PTREYYHRHT RCLYWEGKLI LPFGDQFWFR 361 YLLGWLMPPK VSILLKATOGE AIRNYYFIDMH VIQDMLVPLY 401 KVGDALEWVH REVEVYPIWL CPHKLFKQPI KGQIYPEPGF 441 EYENRQGDTG DAQMYTOVGV YYAPGCVLRG EEFDGLEAVR 481 RMEKWLIENH GFQPQYAVSE LDEKSLWRMF NGELYEECRK 521 KYRAIGTFMS VYYKSKKGRK TEKEVREAEQ AHLETPYPEA $561 \mathrm{D}$
B

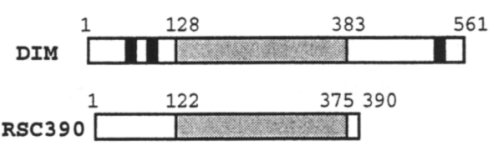

C

DIM $58 \quad$ KRqKehdenvKKviKR1KgR 89 RKpwiavgmRnvdyRRaR 519 RKKyRaigtemsvyYKsKKgRK TGA1A 74 KVIRRlagnReaakRsRIRKK TGA1B 185 KKRaRlVRnResaqlsRqRKK VirD2 417 KRpRedddgepseRKReR N1 534 KRKteeesplKdKdaKK
Figure 5. Structure of the DIM gene product. (A) Predicted amino acid sequence of the DIM protein. $(B)$ Schematic structures of the Arabidopsis DIM protein and the human homolog RSC390. Highly conserved regions between DIM and RSC390 $(\sim 51 \%$ identity) are indicated by shaded boxes. Solid boxes represent bipartite motifs similar to NLSs. (C) Alignment of bipartite motifs present in DIM protein with putative NLSs of other nuclear proteins. The residue numbers for the start of each protein segment are given. Basic residues (Arg and Lys) are in bold, uppercase letters. Sequence information is from the following: for tobacco TGAla and TGAlb, Katagiri et al. (1989); for Agrobacterium VirD2, Howard et al. (1992); for Xenopus N1, Robbins et al. (1991). 
dark-grown seedlings were probed with the longest cDNA of DIM. As shown in Figure 6A, the DIM transcript, which is $\sim 2.0 \mathrm{~kb}$, was detected in both light- and dark-grown wild-type seedlings. No signal was detected in Northern blots with RNA extracted from dim seedlings. This result, in combination with the fact that there is a T-DNA insertion in the $5^{\prime}$ end of the proteincoding sequence, strongly suggests that the T-DNA insertion may have produced a null allele. The analysis of RNA from various organs of Arabidopsis indicated that the DIM gene is constitutively expressed in all organs examined, including roots, rosette leaves, inflorescences, flowers, and siliques (Fig. 6B). Expression of the DIM gene appeared to be unaffected in the de-etiolated mutants, det1 and det2 (Chory 1992), and in a classically identified dwarf mutant, $d w 1$ (Koornneef et al. 1983), as shown in Figure 6C.

An analysis of low stringency Southern hybridizations of Arabidopsis genomic DNA indicates that there are no other genes closely related to DIM (data not shown).

\section{$\operatorname{dim}$ affects the expression of genes involved in cell elongation}

The most dramatic aspect of the dim phenotype is the severe reduction in cell length in numerous tissues. Examination of the epidermal cell morphology of petioles by scanning electron microscopy reveals that mutant cells are markedly shorter and more rounded as compared with wild-type cells (see Fig. 2A,B). Furthermore, microfibrils in a transverse orientation (wild type shown in Fig. 2A), which may be composed of cellulose, are not

A

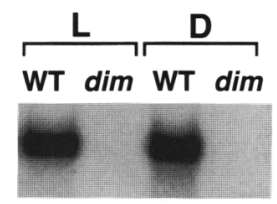

B

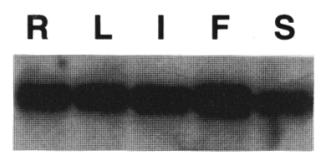

C

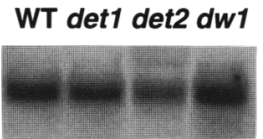

Figure 6. Northern analysis of the DIM mRNA. (A) Total RNA was isolated from 10-day-old light-grown $(\mathrm{L})$ and dark-grown $(\mathrm{D})$ seedlings of wild-type (WT) and dim, and probed with a cDNA fragment of the entire DIM-coding region as described in Materials and methods. $(B)$ RNA was isolated from different parts of wild-type plants, including roots $(R)$, leaves $(L)$, inflorescences (I), flowers (F), and siliques (S). (C) Expression of the DIM gene is unaffected by $\operatorname{det} 1, \operatorname{det} 2$, and $d w 1$ mutations. Total RNA was prepared from 10-day-old light-grown seedlings of wild-type (WT), det1-1 (Chory 1992), det2-1 (Chory 1992), and $d w 1$ (Koornneef et al. 1983).
A

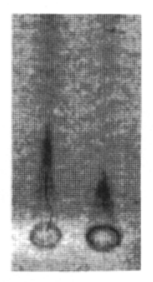

WT $\operatorname{dim}$

B
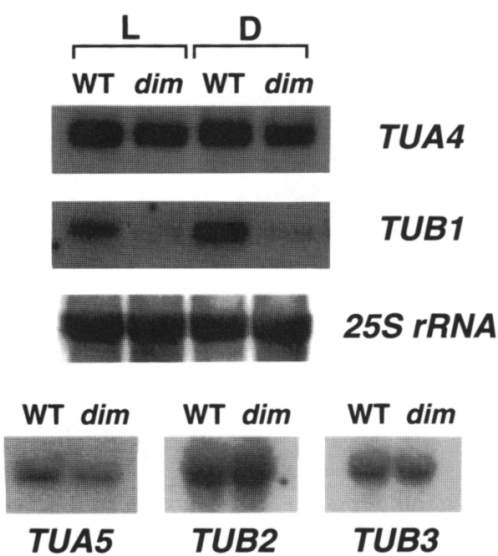

Figure 7. Molecular phenotypes of the dim mutant. $(A)$ Comparison of the level of Yariv reagent-reactive AGPs between wild-type (WT) and dim hypocotyls. Proteins were extracted from hypocotyl sections of 10-day-old dark-grown seedlings and were subjected to rocket gel electrophoresis. Fifty micrograms of protein was loaded in each well. The agarose gel was dried prior to photography. $(B)$ Northern analysis of mRNAs for tubulin genes. Total RNA was isolated from 10-day-old lightgrown (L) and dark-grown (D) seedlings of wild-type (WT) and dim and hybridized with gene-specific probes for tubulin genes. The blot reprobed with the $25 S r R N A$ gene is also shown. For TUA5, TUB2, and TUB3 transcripts, hybridizations with total RNA from 10-day-old light-grown seedlings were shown.

detectable in mutant epidermal cells (Fig. 2B). Recently, we have found in Arabidopsis that hypocotyl length is closely correlated to the amount of arabinogalactan proteins (AGPs) in the hypocotyl (T. Takahashi, J.K. Zhu, and N.-H. Chua, unpubl.). AGPs are proteoglycans present in the extracellular space and plasma membranes, and have been implicated as a cell wall-loosening factor (Zhu et al. 1993). The rocket gel immunoassays shown in Figure 7A demonstrate that the amount of AGPs in dim hypocotyls is lower than that in wild-type hypocotyls.

These results prompted us to examine whether or not the dim mutation leads to the altered expression of genes required for cell elongation. Microtubules are formed by the regulated assembly of $\alpha$ - and $\beta$-tubulins and are thought to play an important role in plant cell elongation. Thus, the levels of expression of tubulin genes were analyzed. Because tubulins are encoded by large multigene families in Arabidopsis (Kopczak et al. 1992; Snustad et al. 1992), Northern hybridizations were performed with gene-specific probes for six $\alpha$-tubulins (TUA1- 
TUA6) and six $\beta$-tubulins (TUB1-TUB6). As shown in Figure $7 \mathrm{~B}$, the dim mutation appeared to decrease the level of the TUB1 mRNA in both light- and dark-grown seedlings (about fourfold less than wild type). It remains to be determined whether the reduced accumulation of TUB1 mRNA reflects the reduced amount of the TUB1 protein or not. In contrast, there was no significant difference in the level of the other tubulin mRNAs between wild-type and dim seedlings (Fig. 7B; data not shown).

\section{Discussion}

We have described a molecular and genetic characterization of the Arabidopsis mutant dim. dim mutants have very short hypocotyls, leaves, stems, and roots and smaller flowers than wild-type plants. Microscopy examination revealed that the decreased stature of the dim plant is the consequence of a severe reduction in cell length. On the basis of our findings presented here, we conclude that the DIM gene product is a novel and crucial component for regulating cell elongation.

Many single-gene mutations conferring a so-called dwarf phenotype have been identified in higher plants since the time of Mendel. Some of them are known to be GA-deficient mutants, which can be restored to the wild-type phenotype by the application of GAs (Koornneef and van der Veen 1980). Mutants with altered sensitivity to auxin also display a pronounced dwarf phenotype (Maher and Martindale 1980; Estelle and Somerville 1987; Timpte et al. 1992). The results of our physiological studies indicated that dim mutants respond to these plant growth regulators in a manner similar to that of wild-type plants. In general, both abscisic acid (ABA) and ethylene also inhibit plant cell elongation. It is unlikely, however, that the signaling pathways for ABA or ethylene are constitutively active in the dim mutant, because the mutant lacks phenotypes expected of such $\mathrm{ABA}$ or ethylene mutants. These phenotypes would include low germination efficiency, accelerated senescence, and the constitutive "triple response" to ethylene /Guzmán and Ecker 1990).

\section{Significance of the DIM gene product in light-grown plants}

The light-grown phenotype of the dim mutant is in contrast to the phenotypes of a series of long hy mutants of Arabidopsis (Koornneef et al. 1980). Whereas dim exhibits extremely short hypocotyls and petioles, and takes longer to flower, hy mutants have elongated hypocotyls and petioles, and show early flowering. These hy mutants are known to have a defect in phytochrome, which functions as the phototransducer for red/far-red reversible reactions, or in blue-light photosensory systems. Physiological studies have revealed that plants have two types of phytochrome, a light-labile species represented by phytochrome A (PHYA), and a light-stable species represented by phytochrome B (PHYB) (for review, see Furuya 1993). Interestingly, the overexpression of PHYA or PHYB induces a short hypocotyl phenotype in trans- genic Arabidopsis plants (Boylan and Quail 1991; Wagner et al. 1991). We have found that dim mutants express wild-type levels of PHYA and PHYB transcripts both in the light and in the dark (data not shown). Therefore, the shortened hypocotyl phenotype of dim cannot be attributed to the accumulation of abnormal levels of phytochrome. Moreover, like wild-type seedlings, dim mutant seedlings show an inhibition of hypocotyl growth under red, far-red, and blue light. Because the dim mutation isolated in this study is most likely a null allele, we conclude that phototransduction pathways for phytochrome and blue light are not impaired by the absence of DIM. However, the function of the DIM gene product may be affected, in part, by light and other stimuli. As shown in Figure 3B, the inhibitory effect of the dim mutation on hypocotyl elongation is much greater in the light than in the dark. Furthermore, the growth of dim roots is blocked less severely than that of the aerial parts of the plants (Table 1). Thus, the DIM gene product appears to be more crucial in light-exposed tissues than in etiolated tissues. Our results can be explained if, in the process of cell elongation, DIM shares the same target|s) with another molecule that is activated by light and represses cell elongation. In the light, the negative effect of such a light-activated molecule on cell elongation would be much stronger in the dim mutant than in the wildtype plant. According to this scenario, the DIM gene product acts as a buffer to the inhibitory effect of light signals on cell elongation. Another possibility that cannot be ruled out is that some components that function in the light, especially during cell elongation (maturation), require the function of the DIM protein, as the ability to elongate is vital to normal light-grown seedling development.

The dim mutation has pleiotropic effects. Electron microscopy of light-grown dim seedlings showed that they have chloroplasts containing a large number of starch grains. Assuming that the inhibition of cell elongation by the dim mutation is associated with a global reduction in cell wall synthesis, the unusual accumulation of starch grains in dim chloroplasts can be interpreted as a result of reduced or delayed sugar metabolism related to wall biogenesis. Delayed flowering may also reflect a secondary effect of reduced sugar metabolism. Mutants deficient in starch degradation have been reported to have a reduced growth rate under a 12 -hr photo period (Caspar et al. 1991).

\section{Effects of the dim mutation on dark-grown seedlings}

A particularly interesting phenotype of the dim mutant is its ability to undergo partial photomorphogenesis in the dark. When grown in complete darkness, the dim seedling develops open cotyledons and primary leaf buds. These traits are reminiscent of de-etiolated mutants, in which light-dependent developmental pathways are constitutively activated in the absence of light (Chory 1992; Deng 1994). However, these morphological changes in the dark-grown dim mutant were accompanied by neither the development of chloroplasts nor the induction 
of light-responsive genes. This observation indicates that the dim mutation uncouples chloroplast development and activation of light-responsive genes from light-dependent morphological changes. In Arabidopsis, de-etiolated phenotypes can be mimicked by growth on solid media supplemented with cytokinins (Chory et al. 1994) or by shaking plants in liquid culture (Rédei et al. 1974). In the latter method, growth from seed to flower can even be accomplished in total darkness. Unlike those cases, dim mutants show only the initial morphological manifestations of de-etiolation on normal media. If the reduction in cell size induced by light is involved in promoting subsequent cell growth, some aspects of the differentiation in dark-grown dim seedlings might be attributable simply to the physical effect of the reduced size in dim cells. It is also possible that changes in the physical and the physiological state of mutant cells affect their ability to respond to phytohormones in shoot apices whose development is primarily under the control of endogenous phytohormones in darkness. Three other photomorphogenic mutants, cop2, cop3 (Hou et al. 1993), and det2 (Chory 1992), and the auxin-resistant mutant axr2 (Timpte et al. 1992) also show partial deetiolation in the dark. The mechanisms that give rise to the phenotype in these mutants remain to be elucidated. We note that in the light, det2 and axr2 mutants also have morphological phenotypes similar to that of $\mathrm{dim}$.

\section{Possible roles of the DIM gene product in cell elongation}

As part of this study, we have cloned and sequenced the DIM gene. The nucleotide sequence predicts a novel 65$\mathrm{kD}$ protein with no significant homology to any known sequence except to an expressed sequence-tag clone isolated from human cells. The strong similarity between a region ( $\sim 255$ residues) in the middle of DIM to a region in the middle of the human protein suggests a common functional domain. The arrangement of basic amino acids in the amino- and carboxy-terminal regions of DIM shows high similarity to the consensus bipartite NLS. This suggests that the DIM protein may be localized in the nucleus. It should be noted that some nonnuclear proteins contain the same motif, and many of these are secreted or targeted to other organelles (Robbins et al. 1991). It is also possible that a hydrophobic domain near the amino terminus is involved in membrane spanning or attachment of the protein. Once antibodies specific for the DIM protein are available, the intracellular localization of DIM will be directly determined by immunoelectron microscopy.

On the basis of the data obtained in this study, there are several possible ways in which DIM could affect cell elongation. The putative NLSs in the DIM protein suggest that DIM is a nuclear protein that may be involved in transcriptional regulation. The fact that TUB1 RNA expression is reduced in dim mutants also suggests that DIM may act directly or indirectly as a transcriptional activator. Tubulins are major components of microtubules, which play vital roles in many aspects of the cell division cycle (for review, see Fosket and Morejohn 1992). Elongating, nondividing cells typically have only the cortical array of microtubules. A recent study of $A r$ abidopsis tubulin genes in our laboratory has revealed that the TUB1 gene is preferentially expressed in elongating dark-grown seedlings (W.-M. Leu and N.-H. Chua, unpubl.|, suggesting that TUB1 expression might be correlated with hypocotyl cell elongation. Interestingly, among the $12 \alpha$-and $\beta$-tubulin genes examined, only the expression of the TUB1 gene is reduced in dim seedlings. Although there is little evidence of microtubule arrayspecific tubulin isotypes in plants (Carpenter et al. 1992), it is possible that TUB1 is specifically involved in the formation of the cortical microtubule array and that its full expression requires the function of the DIM protein. In spite of the reduction of TUB1 mRNA accumulation, however, dim mutants retain the ability to elongate hypocotyls in the dark (Fig. 3B). This result suggests that the dim mutation might negatively affect the expression of some genes other than TUB1, which are required for cell elongation especially in the light. The lack of microfibrils in transverse orientation in the dim mutant (Fig. 2B) might be explained by the reduced expression of those genes. It remains to be determined whether the dim mutation affects the amount of AGPs at the transcriptional level or not.

The process of cell elongation in plants requires loosening of the cell wall structure and the deposition of new material. The plant cell wall is composed of complex polysaccharides and proteins as well as crystalline cellulose microfibrils (for review, see Carpita and Gibeaut 1993|. Thus, DIM could also be a structural cell wall protein or an enzyme required for the loosening and subsequent expansion of the cell wall. An alternative possibility is that in elongating cells, DIM might function in the biosynthesis of cell wall materials or be involved in the transport of wall materials. However, DIM has no homology to any known metabolic enzyme. Furthermore, a highly homologous protein is present in human cells that have no walls. It is also important to note that dark-grown dim seedlings still possess the ability to elongate hypocotyls to some extent.

In conclusion, we believe that DIM plays a critical role in the process of plant cell elongation, although it is clear that other factors also function in this process. Further molecular and biochemical characterization of the dim mutant will not only clarify the mode of action of the DIM protein but may also provide insights into the function of the homologous protein present in human cells.

\section{Materials and methods}

Plant materials and growth

The dim mutant was isolated from lines of Arabidopsis thaliana (ecotype C24) harboring T-DNA insertions, which were generated in our laboratory by using a pMON721-derived vector (Monsanto). This mutant was backcrossed into wild-type Columbia (Col-0) eight times prior to the experiments described here. Mutant alleles, $\operatorname{det} 1-1$ and $\operatorname{det} 2-1$, were kindly provided by J. Chory (Chory 1992). dw1 was obtained from the Arabidopsis Biological Resource Center (Columbus, $\mathrm{OH}$ ). 
Plants were grown to maturity under 16 -hr fluorescent illumination at $20^{\circ} \mathrm{C}$. For seedling growth, seeds were surface sterilized for $3 \mathrm{~min}$ in $95 \%$ bleach (Clorox) and $0.02 \%$ Triton X-100, rinsed twice, and then plated on $0.8 \%$ agar $(\mathrm{pH} 5.5$ ) containing $1 \times$ MS salts $\{\mathrm{GIBCO}\}$ and $3 \%$ sucrose. Plates were kept in the dark at $4^{\circ} \mathrm{C}$ for 2 days before germination.

The number of chloroplasts in leaf mesophyll protoplasts was measured under light microscopy as described by Chory et al. (1989). Chlorophyll determinations were performed on leaves from 15-day-old seedlings (Chory et al. 1989).

\section{Isolation of genomic and cDNA clones}

Genomic DNA flanking the left end of the T-DNA insert was isolated by inverse PCR as follows. DNA from homozygous dim seedlings was prepared as described by Takahashi and Komeda (1989), and $1 \mu \mathrm{g}$ was digested with BamHI and BgIII restriction enzymes. The DNA was ligated at $16^{\circ} \mathrm{C}$ in a $200-\mu l$ reaction, giving a final concentration of $1 \times$ ligation buffer (Boehringer Mannheim), $1 \mathrm{~mm}$ ATP, and 10 units of T4 DNA ligase (Boehringer Mannheim). The ligation mixture was extracted with phenol- $\mathrm{CHCl}_{3}$ and with $\mathrm{CHCl}_{3}$, and DNA was precipitated with ethanol. The DNA was then added to a PCR mixture (Gasch et al. 1992). PCR conditions were 30 cycles at $94^{\circ} \mathrm{C}$ for 1 $\mathrm{min}, 58^{\circ} \mathrm{C}$ for $1.5 \mathrm{~min}$, and $72^{\circ} \mathrm{C}$ for $2.5 \mathrm{~min}$. Synthetic oligonucleotides corresponding to regions between the internal BamHI site and the breakpoint of the T-DNA, which were determined by genomic Southern analysis, were used as primers.

PCR products were cloned into the pBluescript vector (Stratagene) and used to screen an Arabidopsis Columbia wild-type genomic library (Clontech). Restriction fragments of genomic clones isolated from the library were subcloned into pBluescript. cDNA clones were isolated from a $\lambda Z A P$ cDNA library (Gasch et al. 1990) by using a 2.5-kb BamHI fragment of genomic DNA containing the DIM-coding region as a probe. DNA sequencing was performed by using Taq DNA polymerase, dye primers, and a $373 \mathrm{~A}$ automated DNA sequencer as recommended by the manufacturer (ABI). Standard molecular techniques were from Sambrook et al. (1989).

\section{Northern blot analysis}

Total RNA was isolated and separated on $1.2 \%$ agarose gels, as described previously (Takahashi and Komeda 1989). The RNA was blotted to Zeta-probe membranes (Bio-Rad) and hybridized to random primer-labeled probes according to the manufacturer's instructions. Gene-specific probes for tubulins (TUAlTUA6, TUB1-TUB6) were supplied by Dr. C.D. Silflow (Kopczak et al. 1992; Snustad et al. 1992). The CAB1 cDNA clone was obtained from R. Mache (Université Joseph Fourier, Grenoble, France). The probe for $R B C L$ was provided by D.A. Christopher (Texas A\&M University, College Station). The genomic clone of the CHS gene was a gift from F.M. Ausubel (Feinbaum and Ausubel 1988). For control hybridizations, all blots were also probed with the EcoRI fragment from pTT25 /genomic clone for $25 S$ rRNA) (T. Takahashi, unpubl.). Relative amounts of $\mathrm{mR}$ NAs were determined by scanning autoradiograms with the Adobe Photoshop program (Adobe Systems Inc.).

\section{Mapping of the DIM locus}

CAPS mapping was carried out as described (Konieczny and Ausubel 1993). We crossed the original dim mutant (ecotype C24) with two wild-type ecotypes, Columbia and Landsberg erecta. From each of the crosses, $F_{2}$ individual plants with the dim phenotype were selected for CAPS analysis. PCR primers were purchased from Research Genetics. Genetic map distances were calculated as described by Koornneef and Stam (1992).

\section{Complementation}

The plant transformation vector pTT68 was constructed as follows. An EcoRI-SmaI fragment of pTT16 (Takahashi and Komeda 1989) containing the cauliflower mosaic virus 35S promoter and the Escherichia coli hygromycin B phosphotransferase $(H P T)$ gene was cloned into pBluescript. The $35 S-H P T$ fusion gene was then transferred as a HindIII-BamHI fragment into a binary vector VIP50 (kindly provided by A.R. van der Krol, Agricultural University, Wageningen, The Netherlands) derived from pMON865 (Monsanto). A 4-kb genomic fragment, from $0.9 \mathrm{~kb}$ upstream of the DIM start codon to $1.1 \mathrm{~kb}$ downstream of the stop codon (Fig. 5B) was then cloned into the HindIII site of the vector to generate pTT68. Plant transformation was performed as described by Valvekens et al. (1988). Regenerants grown in the presence of $20 \mathrm{mg} /$ liter of hygromycin were selected for crossing with dim heterozygous plants for the complementation test.

\section{Arabinogalactan protein assays}

Hypocotyls of 10-day-old dark-grown seedlings were homogenized in Eppendorf tubes in the presence of extraction buffer 10.1 $\mathrm{M}$ Tris- $\mathrm{HCl}, 5 \%$ Triton $\mathrm{X}-100$ at $\mathrm{pH} 8.0$ ) and centrifuged to remove debris, and the extract used for rocket gel electrophoresis according to a method described previously (Zhu et al. 1993). Arabinogalactan proteins were detected by $\beta$-glucosyl Yariv reagent (Komalavilas et al. 1991). Protein assays were performed using the Bradford reagent (Bio-Rad).

\section{Microscopy}

Samples for light microscopy were fixed overnight in a solution containing $50 \%$ ethanol, $5 \%$ acetic acid, and $4 \%$ formaldehyde and solidified in paraplast after gradual dehydration. Sections 6 $\mu \mathrm{m}$ thick were stained with toluidine blue. For transmission electron microscopy, tissues were fixed with $4 \%$ paraformaldehyde, $5 \%$ glutaraldehyde, and $0.1 \mathrm{M} \mathrm{CaCl}_{2}$ in $0.1 \mathrm{M}$ cacodylate buffer ( $\mathrm{pH} \mathrm{7.2)} \mathrm{on} \mathrm{ice} \mathrm{for} 3 \mathrm{hr}$, rinsed with the same buffer, and postfixed with the buffer containing $1 \%$ osmium tetroxide for 2 hr. Samples were then dehydrated with an ethanol series and propylene oxide, and embedded in Epon-Araldite. Specimens were observed in a JEOL 1200EX microscope (Akishima, Japan). Scanning electron microscopy for petiole epidermis was carried out on tissue fixed and dehydrated as described above, and critical point dried in liquid $\mathrm{CO}_{2}$. Samples were coated with gold. Dark-grown seedlings were snap frozen in liquid nitrogen and examined in a frozen hydrated state without coating by low vacuum scanning electron microscopy.

\section{Acknowledgments}

We are especially grateful to Ulrich Klahre and Diane Shevell for many helpful discussions. We also thank John Mundy for careful reading of the manuscript, and Wei-Ming Leu for sharing unpublished results. The expert technical assistance of ChunHai Dong and Xiang Ling Cao is greatly appreciated. We thank Drs. F.M. Ausubel, J. Chory, D.A. Christopher, R. Mache, E.A. Nothnagel, C.D. Silflow, and A.R. van der Krol for generously providing materials. T.T. was supported by a fellowship from the Toyobo Biotechnology Foundation of Japan. This work was supported by National Institutes of Health grant GM44640 to N.-H.C. 
The publication costs of this article were defrayed in part by payment of page charges. This article must therefore be hereby marked "advertisement" in accordance with 18 USC section 1734 solely to indicate this fact.

\section{Note added in proof}

The sequence data reported in this paper have been deposited to the GenBank data library under accession number L38520.

\section{References}

Bowler, C., G. Neuhaus, H. Yamagata, and N.-H. Chua. 1994. Cyclic GMP and calcium mediate phytochrome phototransduction. Cell 77: 73-81.

Boylan, M.T. and P.H. Quail. 1991. Phytochrome A overexpression inhibits hypocotyl elongation in transgenic Arabidopsis. Proc. Natl. Acad. Sci. 88: 10806-10810.

Carpenter, J.L., S.E. Ploense, D.P. Snustad, and C.D. Silflow. 1992. Preferential expression of an $\alpha$-tubulin gene of Arabidopsis in pollen. Plant Cell 4: 557-571.

Carpita, N.C. and D.M. Gibeaut. 1993. Structural models of primary cell walls in flowering plants: Consistency of molecular structure with the physical properties of the walls during growth. Plant J. 3: 1-30.

Caspar, T., T.-P. Lin, G. Kakefuda, L. Benbow, J. Preiss, and C. Somerville. 1991. Mutants of Arabidopsis with altered regulation of starch degradation. Plant Physiol. 95: 1181-1188.

Chory, J. 1992. A genetic model for light-regulated seedling development in Arabidopsis. Development 115: 337-354.

Chory, J., C.A. Peto, M. Ashbaugh, R. Saganich, L. Pratt, and F. Ausubel. 1989. Different roles for phytochrome in etiolated and green plants deduced from characterization of Arabidopsis thaliana mutants. Plant Cell 1: 867-880.

Chory, J., D. Reinecke, S. Sim, T. Washburn, and M. Brenner. 1994. A role for cytokinins in de-etiolation in Arabidopsis. Plant Physiol. 104: 339-347.

Cosgrove, D. 1986. Biophysical control of plant cell growth. Annu. Rev. Plant Physiol. Plant Mol. Biol. 37: 377-405.

Deng, X.-W. 1994. Fresh view of light signal transduction in plants. Cell 76: 423-426.

Devlin, P.F., S.B. Rood, D.E. Somers, P.H. Quail, and G.C. Whitelam. 1992. Photophysiology of the elongated internode (ein) mutant of Brassica rapa: ein mutant lacks a detectable phytochrome B-like polypeptide. Plant Physiol. 100: 14421447.

Estelle, M.A. and C. Somerville. 1987. Auxin-resistant mutants of Arabidopsis thaliana with an altered morphology. Mol. \& Gen. Genet. 206: 200-206.

Feinbaum, R.L. and F.M. Ausubel. 1988. Transcriptional regulation of the Arabidopsis thaliana chalcone synthase gene. Mol. \& Cell. Biol. 8: 1985-1992.

Finkelstein, R., M. Estelle, J. Martinez-Zapater, and C. Somerville. 1987. Arabidopsis as a tool for the identification of genes involved in plant development. In Plant gene research: Temporal and spatial regulation of plant genes (ed. D.P.S. Verma and R. Goldberg), Vol. 5, pp. 1-25. Springer-Verlag, New York.

Fosket, D.E. and L.C. Morejohn. 1992. Structural and functional organization of tubulin. Annu. Rev. Plant Physiol. Plant Mol. Biol. 43: 201-240.

Furuya, M. 1993. Phytochromes: Their molecular species, gene families, and functions. Annu. Rev. Plant Physiol. Plant Mol. Biol. 44: 617-645.

Gasch, A., A. Hoffmann, M. Horikoshi, R.G. Roeder, and N.-H.
Chua. 1990. Arabidopsis thaliana contains two genes for TFIID. Nature 346: 390-394.

Gasch, A., T. Aoyama, R. Foster, and N.-H. Chua. 1992. Gene isolation with the polymerase chain reaction. In Methods in Arabidopsis research (ed. C. Koncz, N.-H. Chua, and J. Schell), pp. 342 -356. World Scientific, Singapore, Malaysia.

Guzmán, P. and J.R. Ecker. 1990. Exploiting the triple response of Arabidopsis to identify ethylene-related mutants. Plant Cell 2: 513-523.

Hou, Y., A.G. von Arnim, and X.-W. Deng. 1993. A new class of Arabidopsis constitutive photomorphogenic genes involved in regulating cotyledon development. Plant Cell 5: 329-339.

Howard, E.A., J.R. Zupan, V. Citovsky, and P.C. Zambryski. 1992. The VirD2 protein of $A$. tumefaciens contains a C-terminal bipartite nuclear localization signal: Implications for nuclear uptake of DNA in plant cells. Cell 68: 109-118.

Jorgensen, R. 1990. Altered gene expression in plants due to trans interactions between homologous genes. Trends Biotechnol. 8: 340-344.

Katagiri, F., E. Lam, and N.-H. Chua. 1989. Two tobacco DNAbinding proteins with homology to the nuclear factor CREB. Nature 340: 727-730.

Komalavilas, P., J.-K. Zhu, and E.A. Nothnagel. 1991. Arabinogaractan-proteins from the suspension culture medium and plasma membrane of rose cells. J. Biol. Chem. 266: 1595615965.

Konieczny, A. and F.M. Ausubel. 1993. A procedure for mapping Arabidopsis mutations using co-dominant ecotype-specific PCR-based markers. Plant I. 4: 403-410.

Koornneef, M. and P. Stam. 1992. Genetic analysis. In Methods in Arabidopsis research (ed. C. Koncz, N.-H. Chua, and J. Schell), pp. 83-99. World Scientific, Singapore, Malaysia.

Koornneef, M. and J.H. van der Veen. 1980. Induction and analysis of GA sensitive mutants in Arabidopsis thaliana (L.) Heynh. Theor. Appl. Genet. 58: 257-263.

Koornneef, M., E. Rolff, and C.J.P. Spruit. 1980. Genetic control of light inhibited hypocotyl elongation in Arabidopsis thaliana (L.) Heynh. Z. Pflanzenphysiol. 100: 147-160.

Koornneef, M., J. van Eden, C.J. Hanhart, P. Stam, F.J. Braaksma, and W.J. Feenstra. 1983. Linkage map of Arabidopsis thaliana. J. Hered. 74: 265-272.

Kopczak, S.D., N.A. Haas, P.J. Hussey, C.D. Silflow, and D.P. Snustad. 1992. The small genome of Arabidopsis contains at least six expressed $\alpha$-tubulin genes. Plant Cell 4: 539-547.

Liscum, E. and R.P. Hangarter. 1991. Arabidopsis mutants lacking blue-light dependent inhibition of hypocotyl elongation. Plant Cell 3: 685-695.

Maher, E.P. and S.J.B. Martindale. 1980. Mutants of Arabidopsis with altered responses to auxins and gravity. Biochem. Genet. 18: 1041-1053.

McCormac, A.C., D. Wagner, M.T. Boylan, P.H. Quail, H. Smith, and G.C. Whitelam. 1993. Photoresponses of transgenic Arabidopsis seedlings expressing introduced phytochrome B-encoding cDNAs: Evidence that phytochrome A and phytochrome $B$ have distinct photoregulatory functions. Plant J. 4: 19-27.

McNellis, T.W., A.G. von Arnim, T. Araki, Y. Komeda, S. Miséra, and X.-W. Deng. 1994. Genetic and molecular analysis of an allelic series of cop1 mutants suggests functional roles for the multiple protein domains. Plant Cell 6: 487-500.

Parks, B.M. and P.H. Quail. 1993. hy8, a new class of Arabidopsis long hypocotyl mutants deficient in functional phytochrome A. Plant Cell 5: 39-48.

Pepper, A., T. Delaney, T. Washburn, D. Poole, and J. Chory. 1994. DET1, a negative regulator of light-mediated development and gene expression in Arabidopsis, encodes a novel 
nuclear-localized protein. Cell 78: 109-116.

Rédei, G.P., G. Acedo, and G. Gavazzi. 1974. Flower differentiation in Arabidopsis. Stadler Genet. Symp. 6: 135-168.

Robbins, J., S.M. Dilworth, R.A. Laskey, and C. Dingwall. 1991. Two independent basic domains in nucleoplasmin nuclear targeting sequence: Identification of a class of bipartite nuclear targeting sequence. Cell 64: 615-623.

Rood, S.B., P.H. Williams, D. Pearce, N. Murofushi, L.N. Mander, and R.P. Pharis. 1990. A mutant gene that increases GA production in Brassica. Plant Physiol. 93: 1168-1174.

Sambrook, J., E.F. Fritsch, and T. Maniatis. 1989. Molecular cloning: A laboratory manual. Cold Spring Harbor Labolatory Press, Cold Spring Harbor, New York.

Shibaoka, H. 1994. Plant hormone-induced changes in the orientation of cortical microtubules: Alterations in the crosslinking between microtubules and the plasma membrane. Annu. Rev. Plant Physiol. Plant Mol. Biol. 45: 527-544.

Snustad, D.P., N.A. Haas, S.D. Kopczak, and C.D. Silflow. 1992. The small genome of Arabidopsis contains at least nine expressed $\beta$-tubulin genes. Plant Cell 4: 549-556.

Steeves, T.A. and I.M. Sussex. 1989. Patterns in plant development. Cambridge University Press, Cambridge, UK.

Takahashi, T. and Y. Komeda. 1989. Characterization of two genes encoding small heat-shock proteins in Arabidopsis thaliana. Mol. \& Gen. Genet. 219: 365-372.

Timpte, C.S., A.K. Wilson, and M. Estelle. 1992. Effects of the axr2 mutation of Arabidopsis on cell shape in hypocotyl and inflorescence. Planta 188: 271-278.

Valvekens, D., M. van Montagu, and M. van Lijsebettens. 1988. Agrobacterium tumefaciens-mediated transformation of $A r$ abidopsis thaliana root explants by using kanamycin selection. Proc. Natl. Acad. Sci. 85: 5536-5540.

Wagner, D., J.M. Teppermen, and P.H. Quail. 1991. Overexpression of Phytochrome B induces a short hypocotyl phenotype in transgenic Arabidopsis. Plant Cell 3: 1275-1288.

Zhu, J.K., R.A. Bressan, and P.M. Hasegawa. 1993. Loss of arabinogalactan-proteins from the plasma membrane of $\mathrm{NaCl}$ adapted tobacco cells. Planta 190: 221-226. 


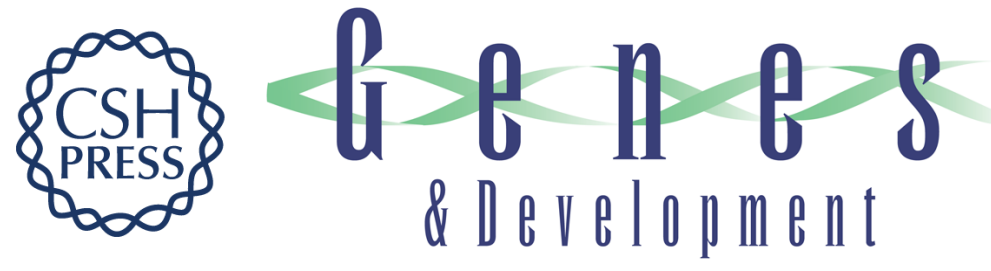

\section{The DIMINUTO gene of Arabidopsis is involved in regulating cell elongation.}

T Takahashi, A Gasch, N Nishizawa, et al.

Genes Dev. 1995, 9:

Access the most recent version at doi:10.1101/gad.9.1.97

References This article cites 43 articles, 19 of which can be accessed free at:

http://genesdev.cshlp.org/content/9/1/97.full.html\#ref-list-1

License

Email Alerting

Service

Receive free email alerts when new articles cite this article - sign up in the box at the top right corner of the article or click here.

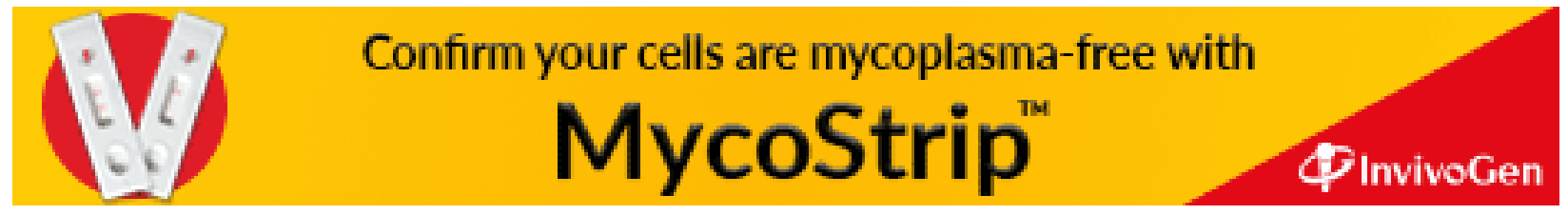

